\title{
Design of Initial Value Control for Modified Lorenz-Stenflo System
}

\author{
Yuan-Long Wu, ${ }^{1}$ Cheng-Hsiung Yang, ${ }^{1}$ and Chang-Hsi Wu ${ }^{2}$ \\ ${ }^{1}$ Graduate Institute of Automation and Control, National Taiwan University of Science and Technology, \\ No. 43, Section 4, Keelung Road, Taipei 106, Taiwan \\ ${ }^{2}$ Department of Electronic Engineering, Lunghwa University of Science and Technology, Taoyuan 333, Taiwan
}

Correspondence should be addressed to Yuan-Long Wu; ylwu@cht.com.tw

Received 20 March 2017; Revised 10 May 2017; Accepted 31 May 2017; Published 5 July 2017

Academic Editor: R. Aguilar-López

Copyright (C) 2017 Yuan-Long Wu et al. This is an open access article distributed under the Creative Commons Attribution License, which permits unrestricted use, distribution, and reproduction in any medium, provided the original work is properly cited.

\begin{abstract}
For the sake of complexity, unpredictability, and exceeding sensitivity to initial conditions in the chaotic systems, there were many studies for information encryption of chaotic systems in recent years. Enhancing the security in information encryption of chaotic systems, an initial value control circuit for chaotic systems is proposed in this paper. By way of changing the initial value, we can change the behavior of chaotic systems and also change the key of information encryption. An analog circuit is implemented to verify the initial value control circuit design.
\end{abstract}

\section{Introduction}

Chaotic systems provide a rich mechanism for signal design and generation, with potential applications to communications and signal processing. Because chaotic signals are typically broadband, noise-like, and difficult to predict, they can be used in various contexts for masking information bearing waveforms. They can also be used as modulating waveforms in spread spectrum systems. This can be useful in many practical circumstances like securing communication channels [1-3], masking signals [4-6], and spreading data sequence or for generating random signals [7]. Four-dimensional LorenzStenflo system is a famous four-dimensional chaotic system; there were many recent works on it [8-11].

The cryptographic system is composed of plaintext, ciphertext, encryption algorithm, key, and decryption algorithm. Dynamic update of the key can reduce its probability of being correctly guessed and improve the security of cryptographic system. In this paper, we introduce an initial value control circuit design for the modified fourdimensional Lorenz-Stenflo system. Furthermore, an analog circuit of the chaotic system is implemented to verify the initial value control circuit design. By way of changing the initial value, we can change the behavior of chaotic systems and also change the key of information encryption.
This paper is organized as follows. In Section 2, nonlinear dynamic characteristics of the modified four-dimensional Lorenz-Stenflo system are analyzed. In Section 3, an analog circuit of the modified chaotic system is implemented to verify the initial value control circuit design. Finally, some concluding remarks are given in Section 4.

\section{Dynamic Analysis of a New Chaotic System}

2.1. Chaotic Equations and Phase Portraits. The LorenzStenflo system is described as $[12,13]$

$$
\begin{aligned}
& \dot{x}_{1}=a x_{2}-a x_{1}+b x_{4}, \\
& \dot{x}_{2}=c x_{1}-x_{1} x_{3}-x_{2}, \\
& \dot{x}_{3}=x_{1} x_{2}-d x_{3}, \\
& \dot{x}_{4}=-x_{1}-a x_{4},
\end{aligned}
$$

where $x=\left[x_{1}, x_{2}, x_{3}, x_{4}\right]^{T}, a$ is Prandtl number, $b$ is rotation number, $c$ is Rayleigh number, and $d$ is geometric parameter. Because four-dimensional Lorenz-Stenflo system is a famous four-dimensional chaotic system, we modify fourdimensional Lorenz-Stenflo system as a study example. We 


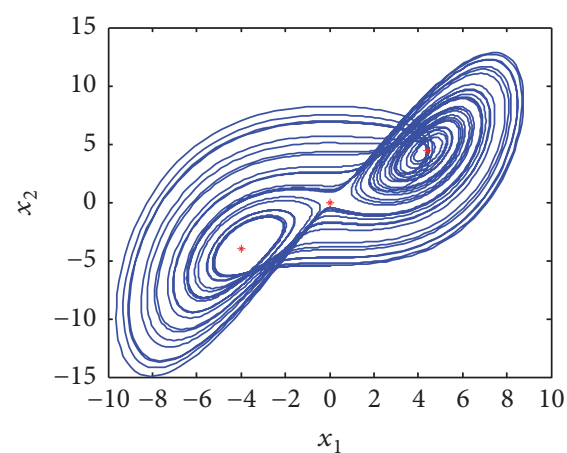

(a)

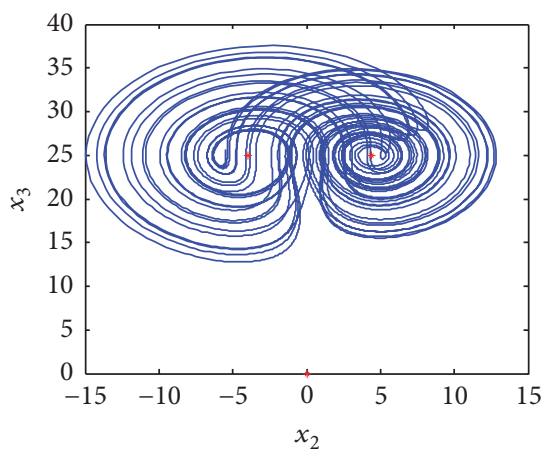

(d)

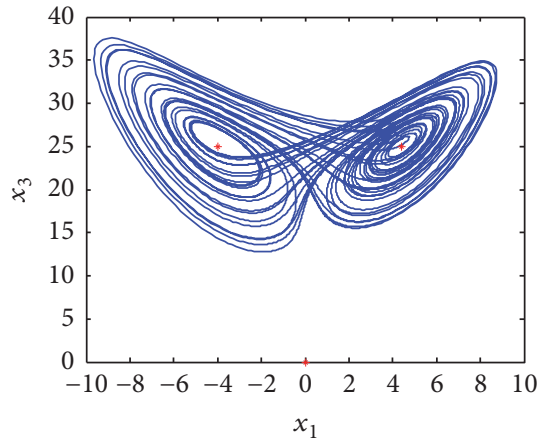

(b)

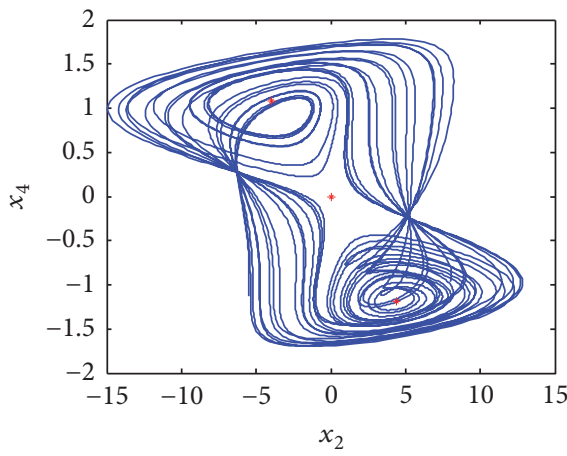

(e)

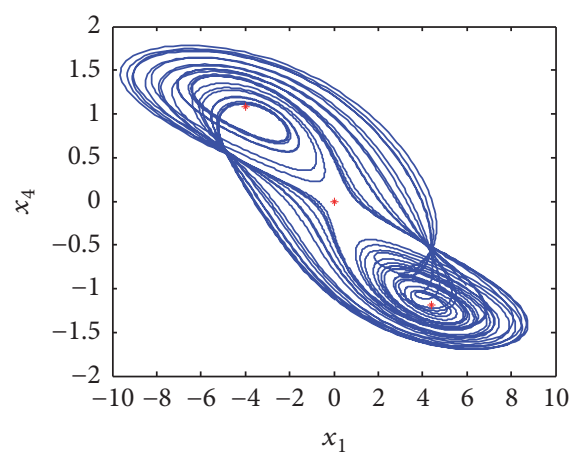

(c)

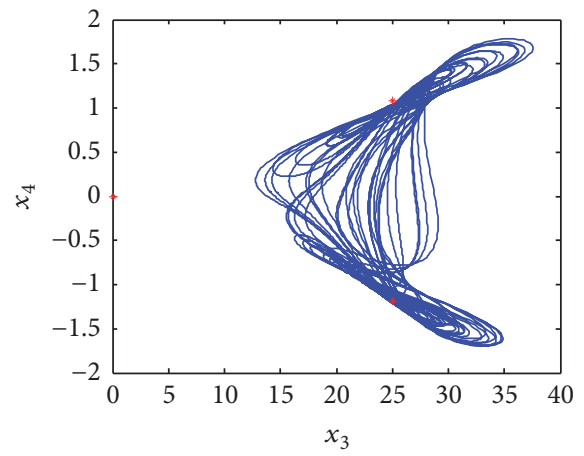

(f)

FIgURE 1: Two-dimensional phase portraits of system equations (2).

modify system equations (1) where new system equations (2) still keep chaotic characteristics [14].

$$
\begin{aligned}
& \dot{x}_{1}=a x_{2}-a x_{1}, \\
& \dot{x}_{2}=c x_{1}-x_{1} x_{3}, \\
& \dot{x}_{3}=x_{1} x_{2}-d x_{3}+b x_{4}, \\
& \dot{x}_{4}=-x_{1}-a x_{4} .
\end{aligned}
$$

The two-dimensional phase portraits of chaotic system equations (2) are shown in Figure 1, in which symbol "*” denotes equilibrium points, with $\mathrm{EP}_{1}, \mathrm{EP}_{2}$, and $\mathrm{EP}_{3}$, with the parameters $a=3.7, b=1.5, c=25, d=0.7$, and $x_{1}(0)=x_{2}(0)=x_{3}(0)=x_{4}(0)=1$.

2.2. Symmetry and Invariance. New chaotic system equations (2) are invariance under the coordinate transform $\left(x_{1}, x_{2}, x_{3}, x_{4}\right) \rightarrow\left(-x_{1},-x_{2}, x_{3},-x_{4}\right)$; chaotic system equations (2) are rotation symmetrical about the $x_{3}$ axis.

2.3. Dissipativity and Existence of Attractor. The state space of chaotic system equations (2) is four-dimensional. The vector field on the right-hand sides of chaotic system equations (2) is defined by

$$
\Phi(x)=\left[\begin{array}{c}
\Phi_{1}(x) \\
\Phi_{2}(x) \\
\Phi_{3}(x) \\
\Phi_{4}(x)
\end{array}\right]=\left[\begin{array}{c}
a x_{2}-a x_{1} \\
c x_{1}-x_{1} x_{3} \\
x_{1} x_{2}-d x_{3}+b x_{4} \\
-x_{1}-a x_{4}
\end{array}\right] .
$$

The divergence of the vector field $\Phi$ is calculated as

$$
\begin{aligned}
\nabla \Phi(x) & =\frac{\partial \Phi_{1}(x)}{\partial x_{1}}+\frac{\partial \Phi_{2}(x)}{\partial x_{2}}+\frac{\partial \Phi_{3}(x)}{\partial x_{3}}+\frac{\partial \Phi_{4}(x)}{\partial x_{4}} \\
& =-2 a-d .
\end{aligned}
$$

A necessary and sufficient condition for system equations (2) to be dissipative is that the divergence of the vector field $\Phi$ is negative. In (4), it is immediate that system equations (2) are dissipative if and only if $a, d \in R^{+}$with an exponential rate:

$$
\frac{d \Phi}{d t}=e^{-(2 a+d)}
$$

Thus, in dynamical system equations (2), a volume element $\Phi_{0}$ is apparently contracted by the flow into a volume element $\Phi_{0} e^{-(2 a+d+1)}$ in time domain. This means that each volume containing the trajectories of this dynamical system shrinks to zero as time approached infinity 


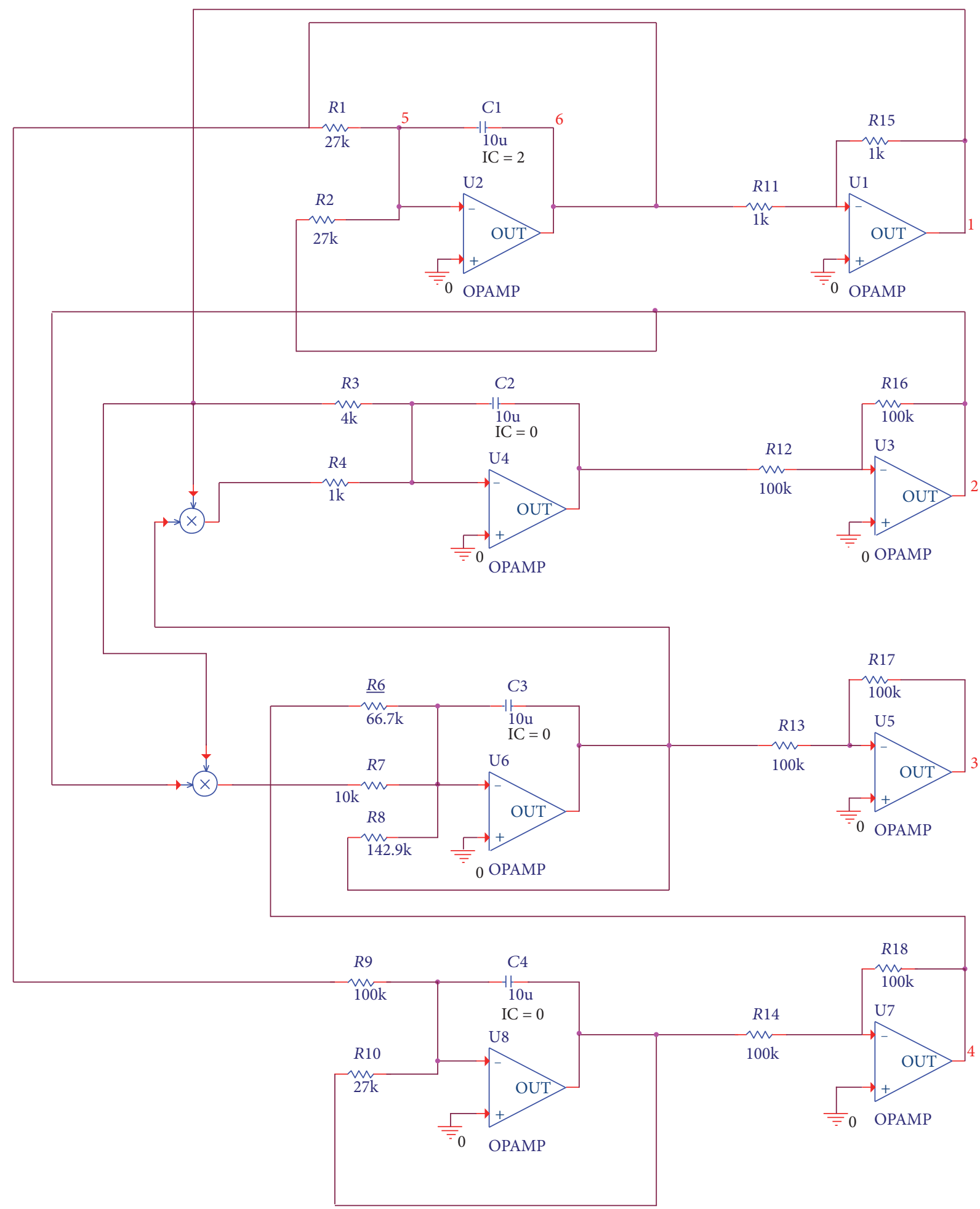

Figure 2: Circuit of chaotic system (2).

at an exponential rate $\nabla \Phi$. So, all the orbits of chaotic system equations (2) will be eventually confined to a special subset that has zero volume, and the asymptotic motion of system equations (2) will settle onto an attractor of the system. Then, the existence of an attractor is proved.
2.4. Equilibrium Points Analysis. The equilibrium points of system equations (2) can be found by solving the following algebraic equations simultaneously:

$$
\begin{aligned}
a x_{2}-a x_{1} & =0, \\
c x_{1}-x_{1} x_{3} & =0,
\end{aligned}
$$




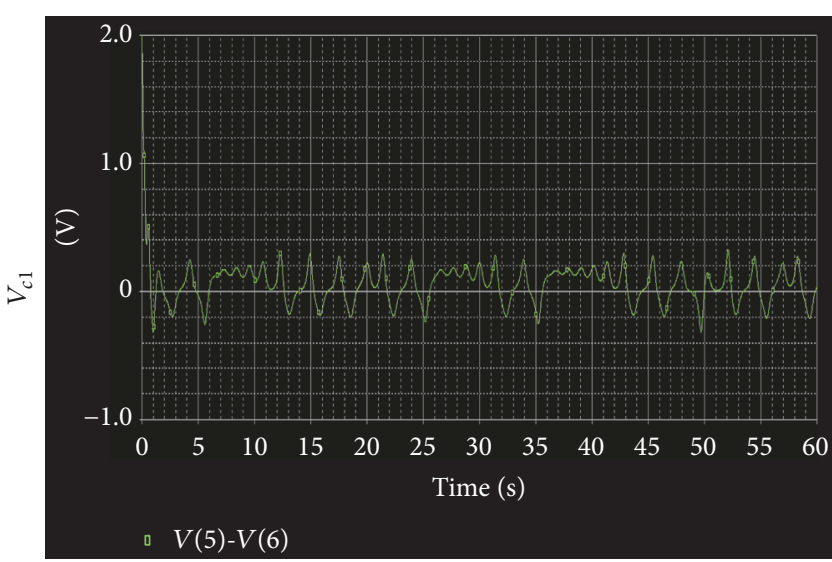

$x_{1}$

(a)

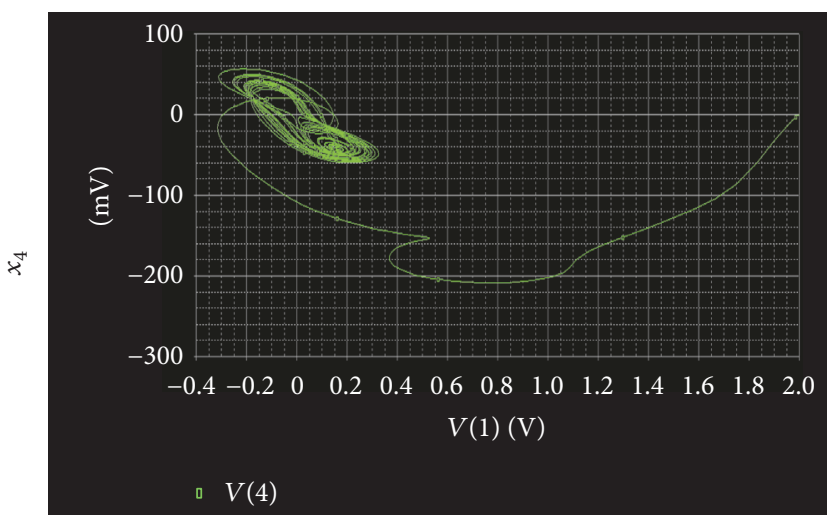

$x_{1}$

(c)

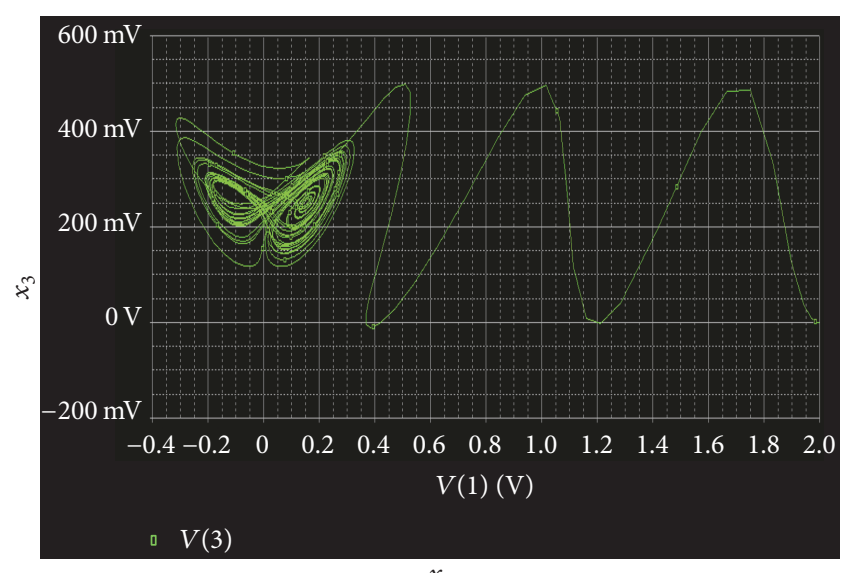

(b)

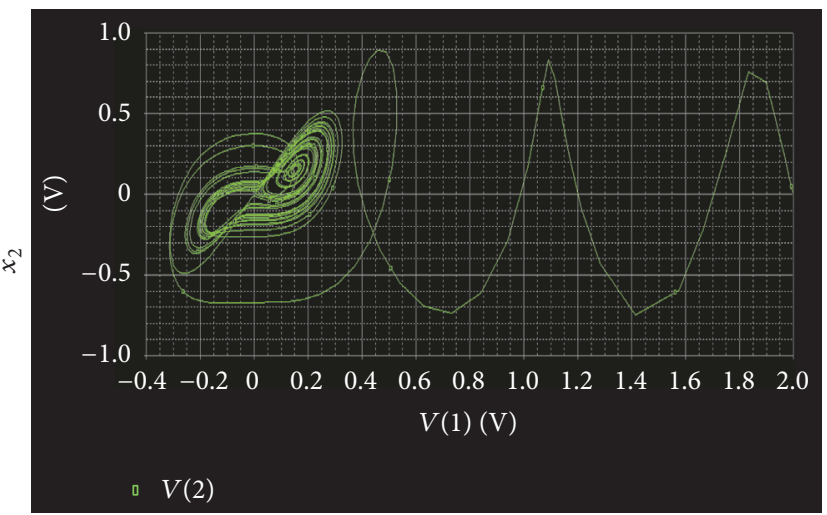

$x_{1}$

(d)

FIGURE 3: Two-dimensional phase portraits of circuit in Figure 2 (default initial value voltage $x_{1}(0)=V_{c 1}=2 \mathrm{~V}$, without initial value control).

$$
\begin{array}{r}
x_{1} x_{2}-d x_{3}+b x_{4}=0, \\
-x_{1}-a x_{4}=0 .
\end{array}
$$

Chaotic system equations (2) have three equilibrium points, respectively, represented as

$$
\begin{aligned}
& \operatorname{EP}_{1}(0,0,0,0), \\
& \operatorname{EP}_{2}\left(\frac{b+\sqrt{4 a^{2} c d+b^{2}}}{2 a}, \frac{b+\sqrt{4 a^{2} c d+b^{2}}}{2 a}, c,\right. \\
& \left.\quad-\frac{b+\sqrt{4 a^{2} c d+b^{2}}}{2 a^{2}}\right), \\
& \operatorname{EP}_{3}\left(\frac{b-\sqrt{4 a^{2} c d+b^{2}}}{2 a}, \frac{b-\sqrt{4 a^{2} c d+b^{2}}}{2 a}, c\right. \\
& \left.\quad-\frac{b-\sqrt{4 a^{2} c d+b^{2}}}{2 a^{2}}\right) .
\end{aligned}
$$

When the parameters are $a=3.7, b=1.5, c=25$, and $d=0.7$, chaotic system equations (2) have three equilibrium points, given by $\mathrm{EP}_{1}(0,0,0), \mathrm{EP}_{2}(4.39091,4.39091,25,-1.18673)$, and $\mathrm{EP}_{3}(-3.98551,-3.98551,25,1.07716)$.

By linearizing chaotic system equations (2), the Jacobian matrix is obtained as

$$
J=\left[\begin{array}{cccc}
-a & a & 0 & 0 \\
c-x_{3} & 0 & -x_{1} & 0 \\
x_{2} & x_{1} & -d & b \\
-1 & 0 & 0 & -a
\end{array}\right]
$$

The Jacobian matrix for chaotic system equations (2) at equilibrium point $\operatorname{EP}_{1}(0,0,0,0)$ is obtained as

$$
J\left(\mathrm{EP}_{1}\right)=\left.\left[\begin{array}{cccc}
-a & a & 0 & 0 \\
c-x_{3} & 0 & -x_{1} & 0 \\
x_{2} & x_{1} & -d & b \\
-1 & 0 & 0 & -a
\end{array}\right]\right|_{x=\mathrm{EP}_{1}}
$$




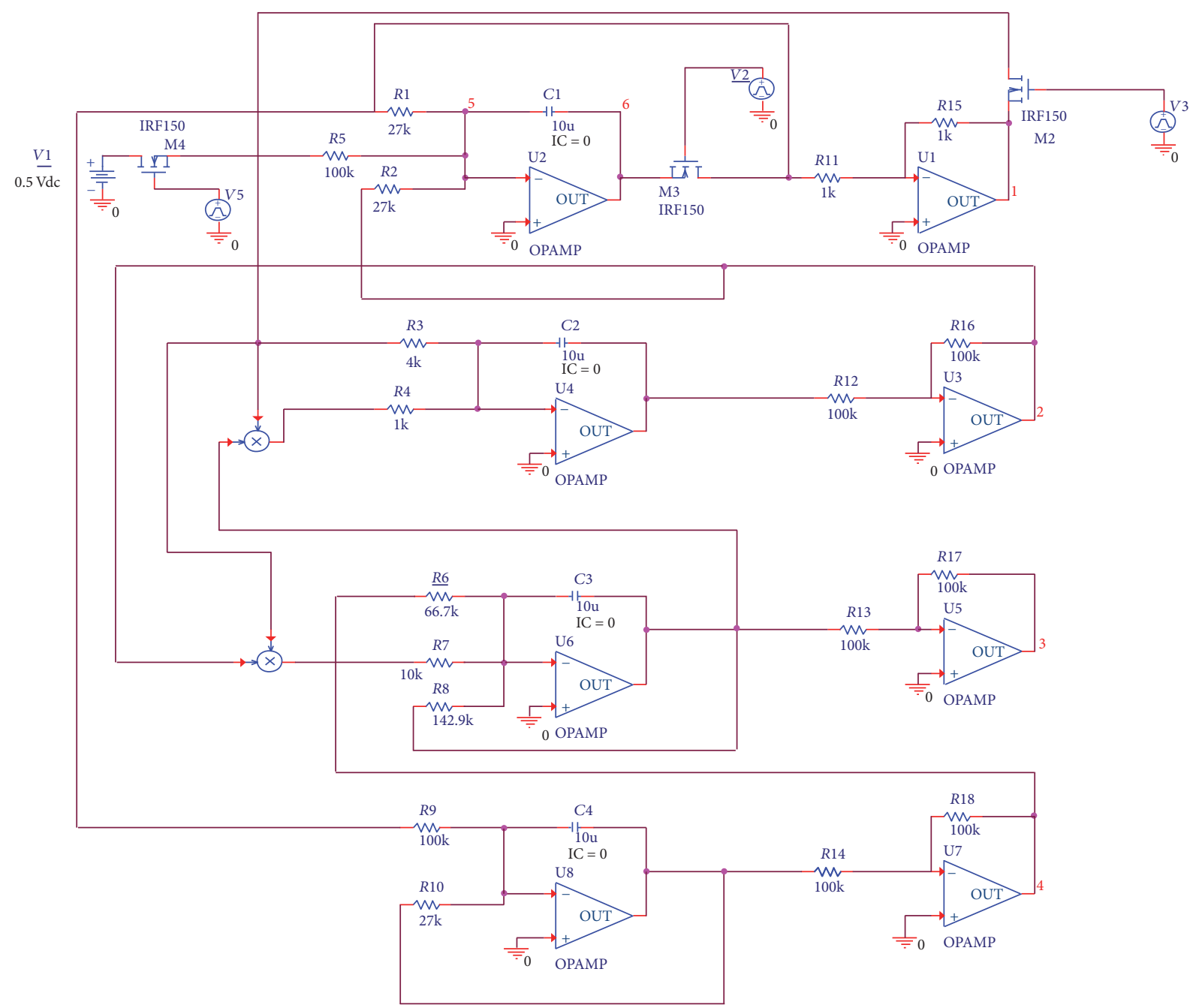

FIGURE 4: Initial value control circuit of chaotic system equations (2).

$$
=\left[\begin{array}{cccc}
-a & a & 0 & 0 \\
c & 0 & 0 & 0 \\
0 & 0 & -d & b \\
-1 & 0 & 0 & -a
\end{array}\right]
$$

(9)

which has the eigenvalues

$$
\begin{aligned}
& \lambda_{1}^{\left(\mathrm{EP}_{1}\right)}=-0.7, \\
& \lambda_{2}^{\left(\mathrm{EP}_{1}\right)}=-3.7, \\
& \lambda_{3}^{\left(\mathrm{EP}_{1}\right)}=-11.6440, \\
& \lambda_{4}^{\left(\mathrm{EP}_{1}\right)}=7.9440 .
\end{aligned}
$$

The Jacobian matrix for chaotic system equations (2) at equilibrium point $\mathrm{EP}_{2}(4.39091,4.39091,25,-1.18673)$ is obtained as

$$
\begin{aligned}
J\left(\mathrm{EP}_{2}\right) & =\left.\left[\begin{array}{cccc}
-a & a & 0 & 0 \\
c-x_{3} & 0 & -x_{1} & 0 \\
x_{2} & x_{1} & -d & b \\
-1 & 0 & 0 & -a
\end{array}\right]\right|_{x=\mathrm{EP}_{2}} \\
& =\left[\begin{array}{cccc}
-a & a & 0 & 0 \\
c-25 & 0 & -4.39091 & 0 \\
4.39091 & 4.39091 & -d & b \\
-1 & 0 & 0 & -a
\end{array}\right]
\end{aligned}
$$




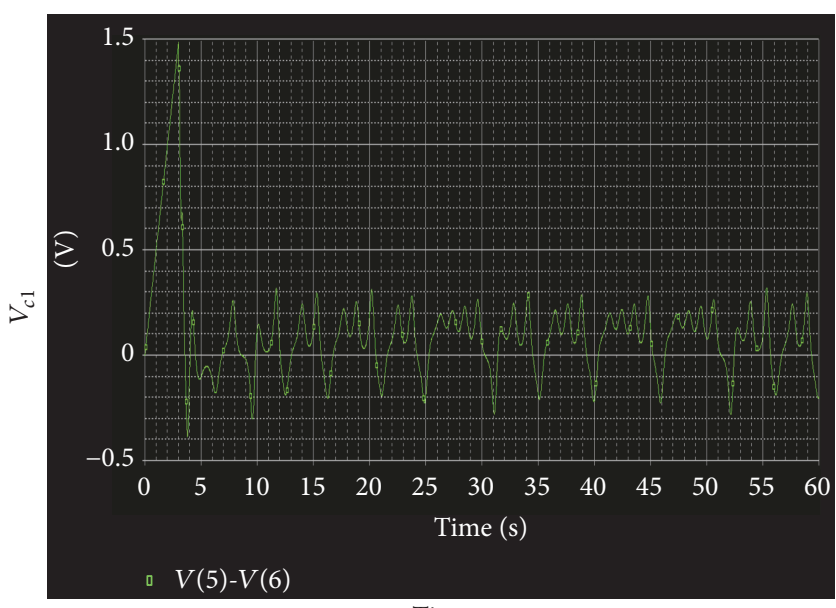

(a)

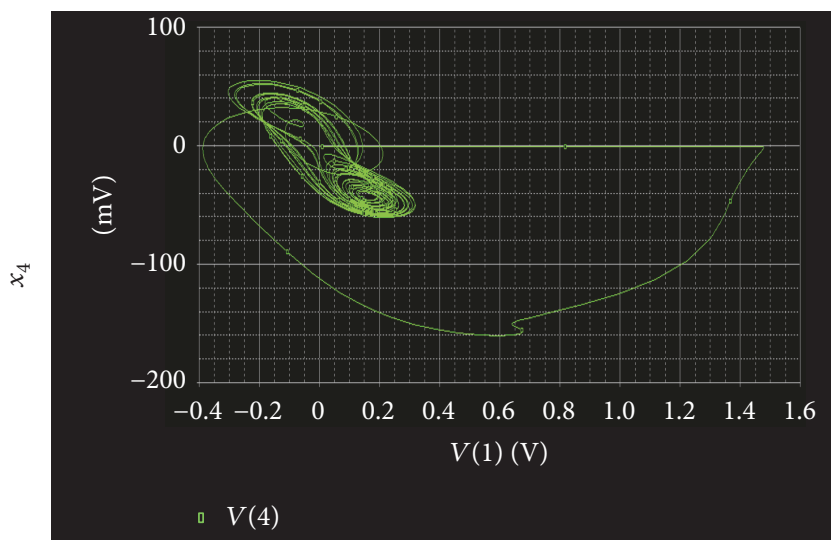

$x_{1}$

(c)

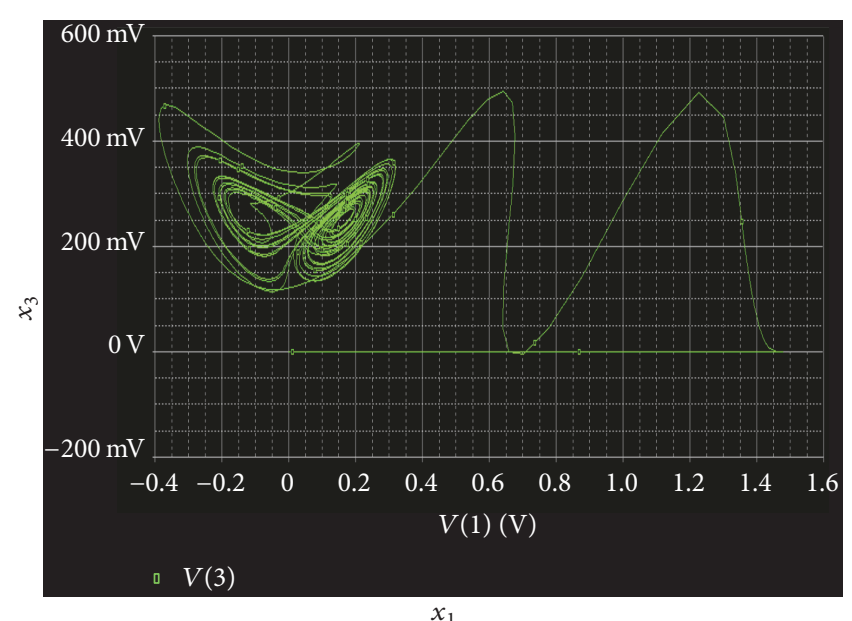

(b)

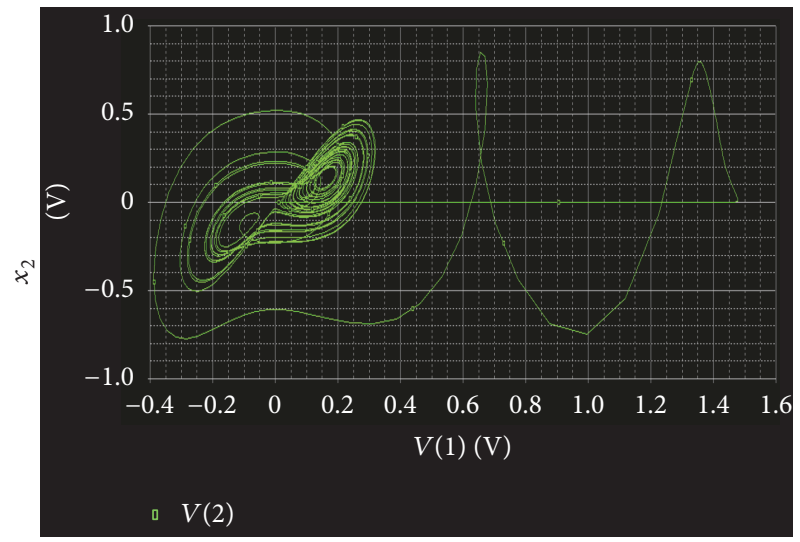

(d)

FIGURE 5: Two-dimensional phase portrait of Figure 4 with control switch turn-on time $3 \mathrm{sec}$ (initial value voltage $x_{1}(0)=V_{c 1}=0.5 \mathrm{~V} * 3 \mathrm{sec}$ $=1.5 \mathrm{~V}$, with initial value control).

which has the eigenvalues

$$
\begin{aligned}
& \lambda_{1}^{\left(\mathrm{EP}_{2}\right)}=0.4161+5.1585 i, \\
& \lambda_{2}^{\left(\mathrm{EP}_{2}\right)}=0.4161-5.1585 i, \\
& \lambda_{3}^{\left(\mathrm{EP}_{2}\right)}=-5.5368, \\
& \lambda_{4}^{\left(\mathrm{EP}_{2}\right)}=-3.3954 .
\end{aligned}
$$

The Jacobian matrix for chaotic system equations (2) at equilibrium point $\mathrm{EP}_{3}(-3.98551,-3.98551,25,1.07716)$ is obtained as

$$
J\left(\mathrm{EP}_{3}\right)=\left.\left[\begin{array}{cccc}
-a & a & 0 & 0 \\
c-x_{3} & 0 & -x_{1} & 0 \\
x_{2} & x_{1} & -d & b \\
-1 & 0 & 0 & -a
\end{array}\right]\right|_{x=\mathrm{EP}_{3}}
$$

$$
=\left[\begin{array}{cccc}
-a & a & 0 & 0 \\
c-25 & 0 & 3.98551 & 0 \\
-3.98551 & -3.98551 & -d & b \\
-1 & 0 & 0 & -a
\end{array}\right]
$$

which has the eigenvalues

$$
\begin{aligned}
& \lambda_{1}^{\left(\mathrm{EP}_{3}\right)}=0.4488+4.7403 i, \\
& \lambda_{2}^{\left(\mathrm{EP}_{3}\right)}=0.4488-4.7403 i, \\
& \lambda_{3}^{\left(\mathrm{EP}_{3}\right)}=-4.7828 \\
& \lambda_{4}^{\left(\mathrm{EP}_{3}\right)}=-4.2148
\end{aligned}
$$

Since the linearization matrices $J\left(\mathrm{EP}_{1}\right), J\left(\mathrm{EP}_{2}\right)$, and $J\left(\mathrm{EP}_{3}\right)$ have at least one eigenvalue with positive real parts, according to Lyapunov stability method [15], then the equilibrium points $\mathrm{EP}_{1}, \mathrm{EP}_{2}$, and $\mathrm{EP}_{3}$ are unstable, and this implies 


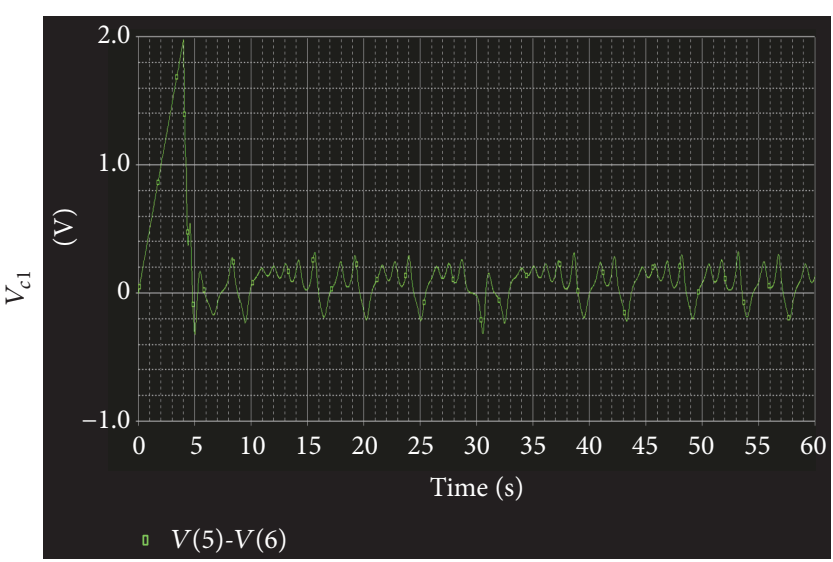

Time

(a)

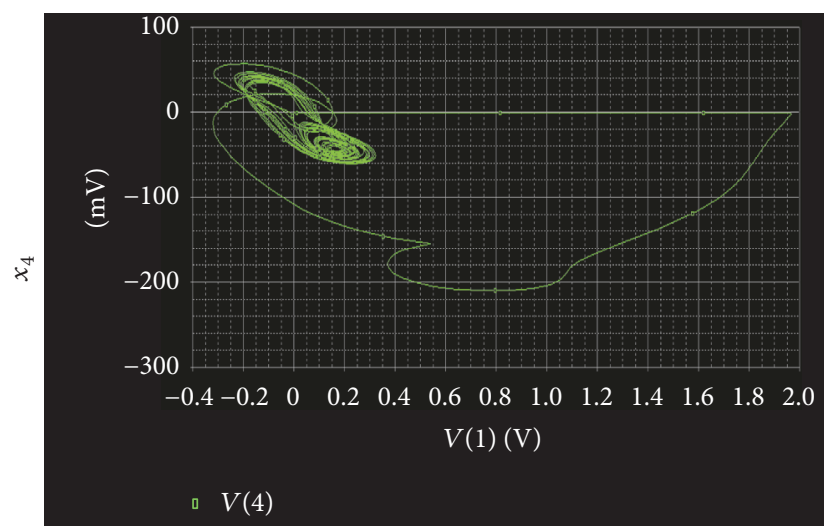

$x_{1}$

(c)

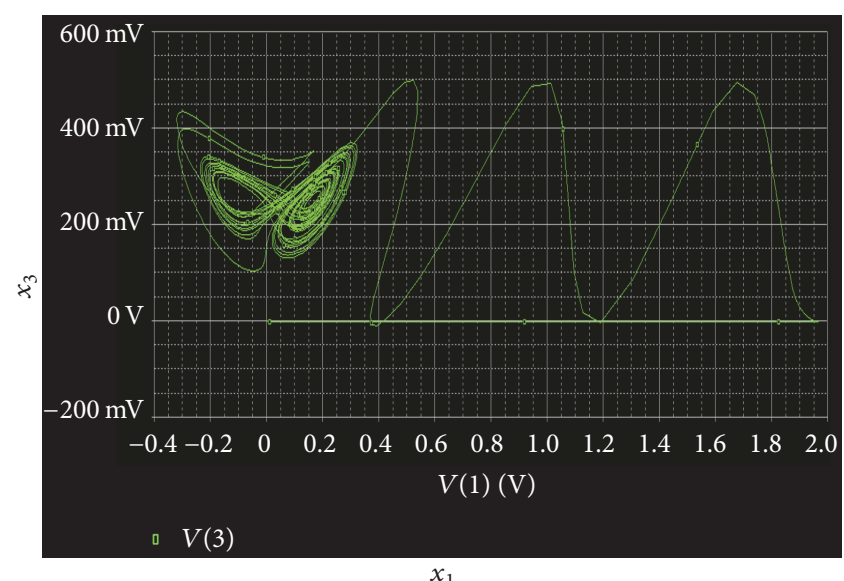

(b)

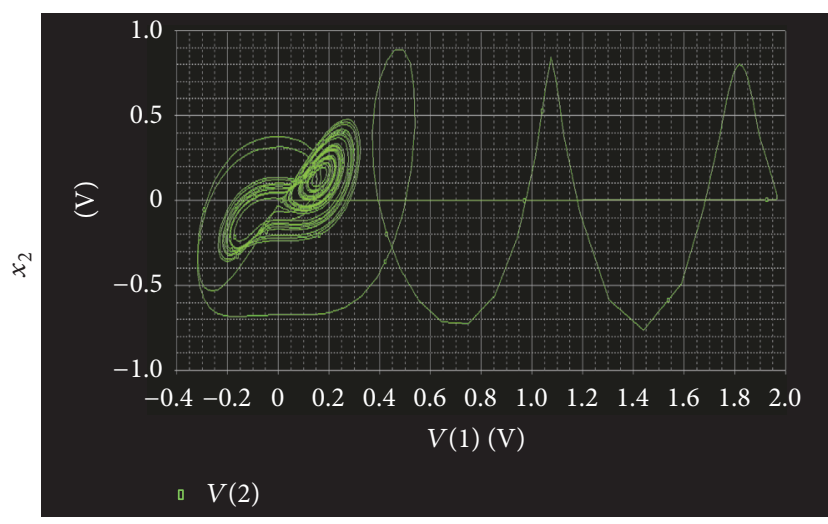

$x^{2}$

FIgURE 6: Two-dimensional phase portrait of Figure 4 with control switch turn-on time $4 \sec$ (initial value voltage $x_{1}(0)=V_{c 1}=0.5 \mathrm{~V} * 4$ sec $=2 \mathrm{~V}$, with initial value control).

chaos in the dissipative chaotic system equation (2). So, the trajectories of chaotic system equation (2) diverge from the three equilibrium points and orbit into the strange attractor of chaotic system Eq. (2).

\section{Initial Value Control Circuit for Chaotic Systems}

The four-dimensional modified Lorenz-Stenflo system can be modeled by an electronic circuit [16-19]. To implement system equations (2), a circuit is constructed in Figure 2; the governing integral equation of the circuit can be written as (15) and some simulation results are showed in Figure 3. A initial value control circuit design for system equations (2) is designed in Figure 4; the two-dimensional phase portraits of chaotic system (2) with initial value control circuit are shown in Figures 5 and 6. From Figures 5 and 6, we can observe the results, when the control switch turn-on time is different, the capacitor is charged to different voltage level. For example, if the control switch turn-on time $=3 \mathrm{sec}$, the capacitor is charged to $V_{c 1}=0.5 \mathrm{~V} * 3 \mathrm{sec}=1.5 \mathrm{~V}$. By way of changing the charged voltage level in capacitor, we can change the initial value in system equations (2) and the behavior of system equations (2) also is modified. The real circuit of the modified four-dimensional Lorenz-Stenflo system is constructed on a breadboard shown in Figure 7. The experimental setup is shown in Figure 8 and the experimental results are shown in Figure 9.

$$
\begin{aligned}
& \dot{x}_{1}=\frac{1}{R_{2} C_{1}} x_{2}-\frac{1}{R_{1} C_{1}} x_{1}, \\
& \dot{x}_{2}=\frac{1}{R_{3} C_{2}} x_{1}-\frac{1}{R_{4} C_{2}} x_{1} x_{3}, \\
& \dot{x}_{3}=\frac{1}{R_{7} C_{3}} x_{1} x_{2}-\frac{1}{R_{8} C_{3}} x_{3}+\frac{1}{R_{6} C_{3}} x_{4}, \\
& \dot{x}_{4}=-\frac{1}{R_{9} C_{4}} x_{1}-\frac{1}{R_{10} C_{4}} x_{4} .
\end{aligned}
$$

\section{Conclusions}

In summary, this paper introduces an initial value control circuit design and nonlinear dynamic analysis of the modified 


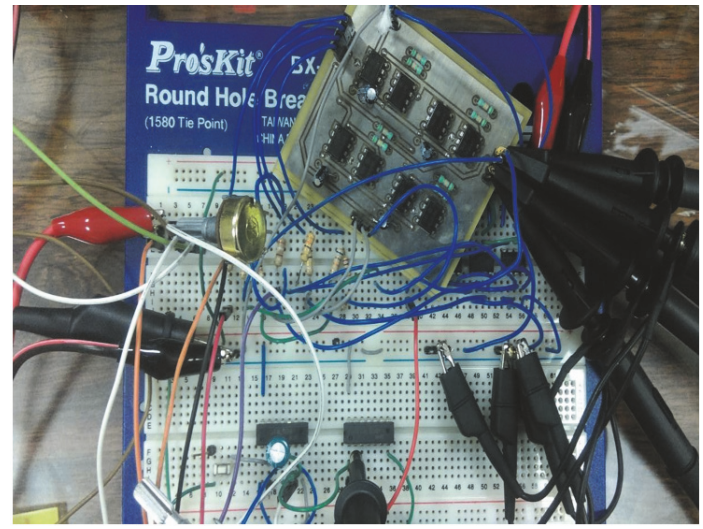

FIGURE 7: Realization circuit of the modified four-dimensional Lorenz-Stenflo system.

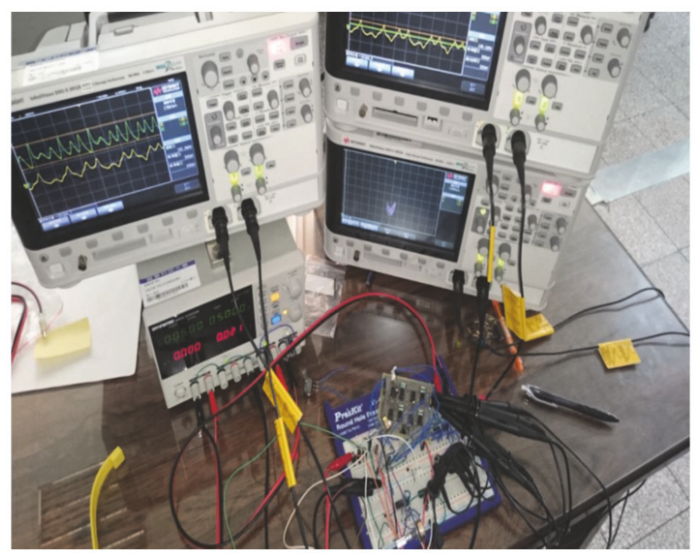

FIGURE 8: Experimental setup for realization of the modified four-dimensional Lorenz-Stenflo system.

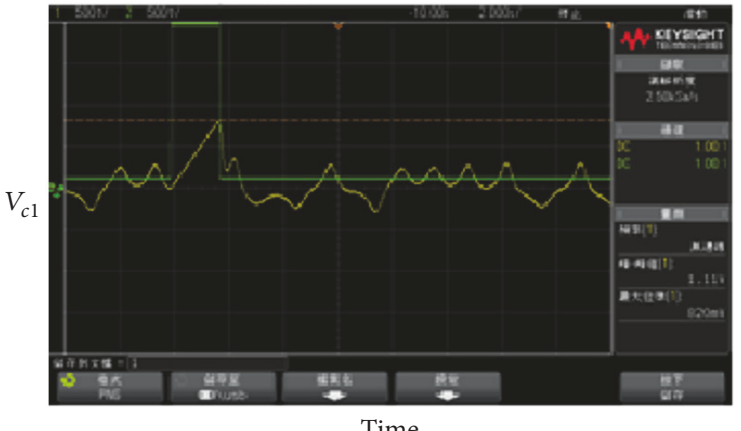

(a)

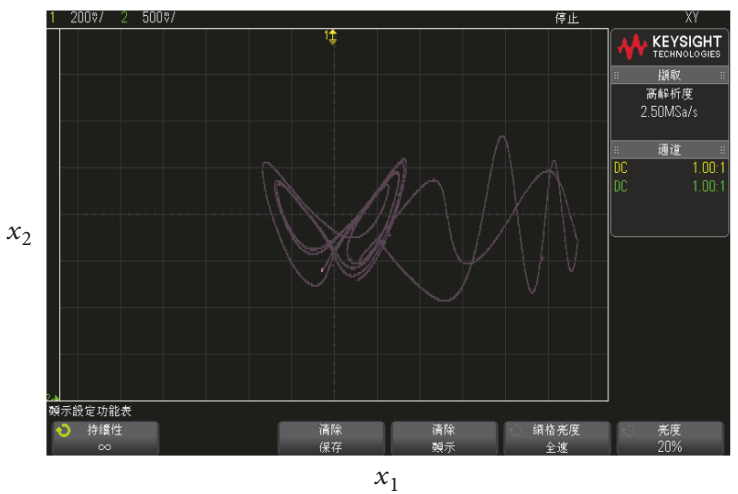

(c)

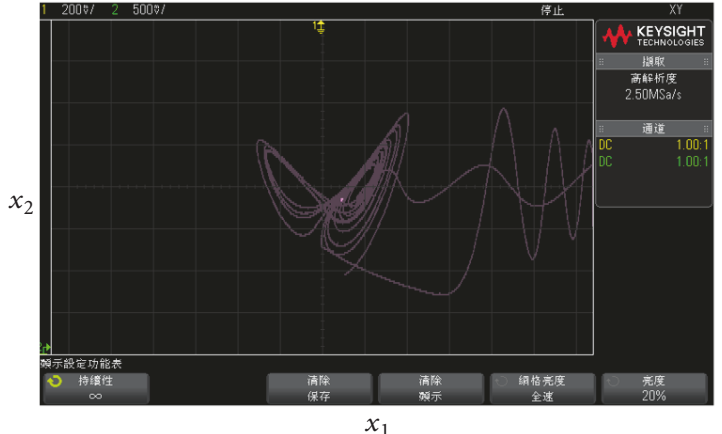

(b)

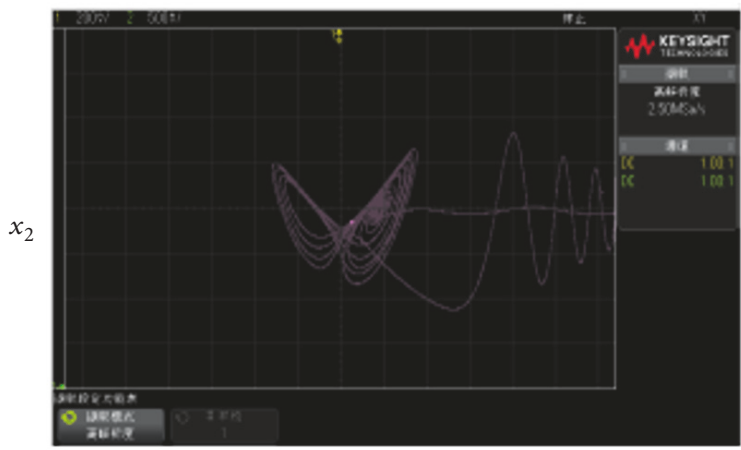

$x_{1}$

(d)

FIGURE 9: Realization circuit results for voltage signals outputs with different control switch turn-on time. 
four-dimensional Lorenz-Stenflo system is presented. Furthermore, an analog circuit of the chaotic system is implemented to verify the initial value control circuit design. We design a charge circuit embedded in modified fourdimensional Lorenz-Stenflo analog circuit and use analog circuit to implement the concept of changing initial values for chaotic systems in mathematics. Because there are operational amplifier (OPA) integrators in the modified fourdimensional Lorenz-Stenflo analog circuit, we only need Metal-Oxide-Semiconductor Field-Effect Transistor (MOSFET) to implement the charge circuit; it is easy to realize. By way of changing the initial value, we can change the behavior of chaotic system and also change the key of information encryption. Next step we will apply the initial value control circuit of chaotic systems for information encryption application.

\section{Conflicts of Interest}

The authors declare that they have no conflicts of interest.

\section{Acknowledgments}

This research was supported by the Ministry of Science and Technology, Taiwan, under Grant no. MOST 104-2221-E-011105.

\section{References}

[1] Z. Li, Y. Soh, and C. Wen, Switched and Impulsive Systems, Analysis, Design, and Applications, vol. 313, Springer, Berlin, Germany, 2005.

[2] I. Pehlivan and Y. Uyaroğlu, "Rikitake attractor and it's synchronization application for secure communication systems," Journal of Applied Sciences, vol. 7, no. 2, pp. 232-236, 2007.

[3] I. Pehlivan and Y. Uyaroğlu, "Simplified chaotic diffusionless Lorentz attractor and its application to secure communication systems," IET Communications, vol. 1, no. 5, pp. 1015-1022, 2007.

[4] P. Galajda and D. Kocur, "Chua's circuit in spread spectrum communication systems," Radioengineering, vol. 11, no. 2, pp. 06-10, 2002.

[5] Y. Uyaroğlu and H. Pehlivan, "Nonlinear Sprott94 case a chaotic equation: synchronization and masking communication applications," Computers and Electrical Engineering, vol. 36, no. 6, pp. 1093-1100, 2010.

[6] I. Pehlivan, Y. Uyaroğlu, and M. Yoğun, "Chaotic oscillator design and realizations of the Rucklidge attractor and its synchronization and masking simulations," Scientific Research and Essays, vol. 5, no. 16, pp. 2210-2219, 2010.

[7] L. Kocarev, Z. Galias, and S. Lian, Eds., Intelligent Computing Based on Chaos, vol. 184 of Studies in Computational Intelligence, Springer, Berlin, Germany, 2009.

[8] S. Pal, B. Sahoo, and S. Poria, "Multistable behaviour of coupled Lorenz-Stenflo systems," Physica Scripta, vol. 8, Article ID 045202, 2014.

[9] S. Pal, B. Sahoo, and S. Poria, "Generalized lag synchronization of delay coupled chaotic systems via linear transformations," Physica Scripta, vol. 87, no. 4, Article ID 045011, 2013.
[10] S. Pal and S. Poria, "Uncertain destination dynamics of delay coupled systems," Physica Scripta, vol. 90, no. 3, Article ID 035203, 2015.

[11] M. Ali Khan and S. Poria, "Projective synchronization of chaotic systems with bidirectional nonlinear coupling," Pramana Journal of Physics, vol. 81, no. 3, pp. 395-406, 2013.

[12] E. N. Lorenz, "Deterministic nonperiodic flow," Journal of the Atmospheric Sciences, vol. 20, pp. 130-141, 1963.

[13] L. Stenflo, "Generalized Lorenz equations for acoustic-gravity waves in the atmosphere," Physica Scripta, vol. 53, no. 1, pp. 8384, 1996.

[14] C.-H. Yang and C.-L. Wu, "Nonlinear dynamic analysis and synchronization of four-dimensional Lorenz-Stenflo system and its circuit experimental implementation," Abstract and Applied Analysis, vol. 2014, Article ID 213694, 17 pages, 2014.

[15] W. Hahn, The Stability of Motion, Springer Verlag, New York, NY, USA, 1967.

[16] X. Wang and G. Chen, "A chaotic system with only one stable equilibrium," Communications in Nonlinear Science and Numerical Simulation, vol. 17, no. 3, pp. 1264-1272, 2012.

[17] Y. Liu, "Circuit implementation and finite-time synchronization of the 4D Rabinovich hyperchaotic system," Nonlinear Dynamics, vol. 67, no. 1, pp. 89-96, 2012.

[18] A. G. Radwan, A. M. Soliman, and A.-L. El-Sedeek, "MOS realization of the double-scroll-like chaotic equation," IEEE Transactions on Circuits and Systems I: Fundamental Theory and Applications, vol. 50, no. 2, pp. 285-288, 2003.

[19] A. Abooee, H. A. Yaghini-Bonabi, and M. R. Jahed-Motlagh, "Analysis and circuitry realization of a novel three-dimensional chaotic system," Communications in Nonlinear Science and Numerical Simulation, vol. 18, no. 5, pp. 1235-1245, 2013. 


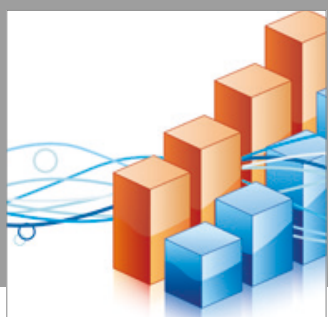

Advances in

Operations Research

vatersals

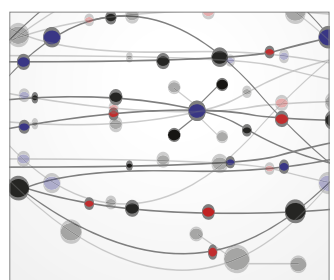

\section{The Scientific} World Journal
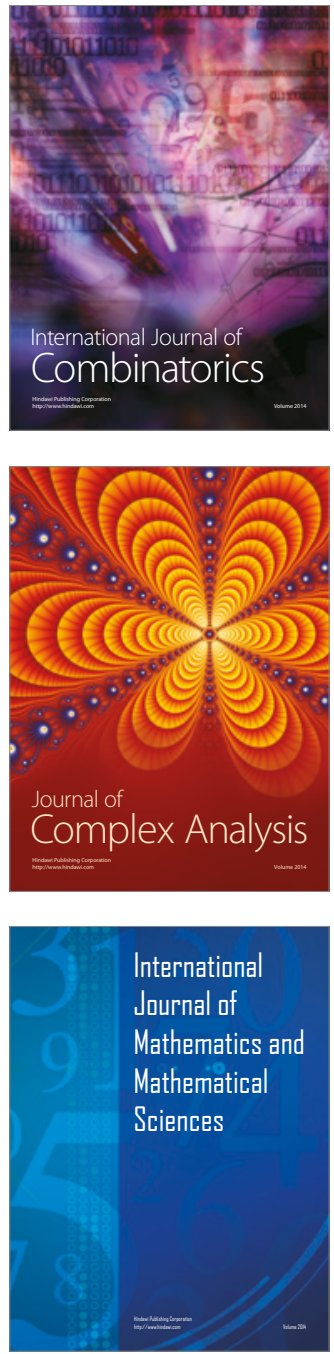
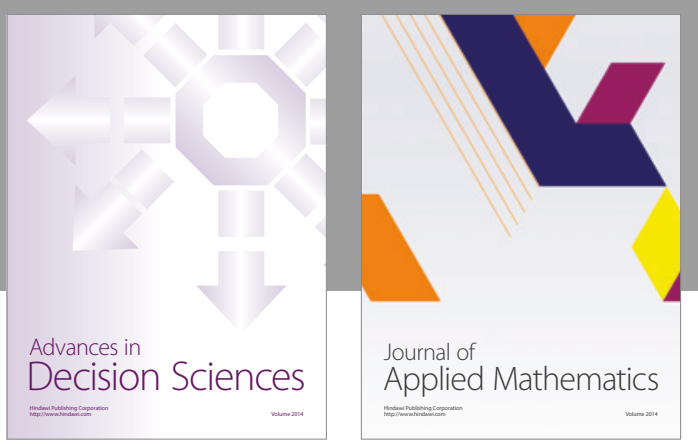

Algebra

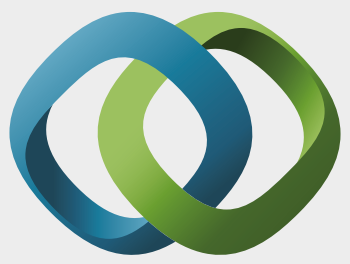

\section{Hindawi}

Submit your manuscripts at

https://www.hindawi.com
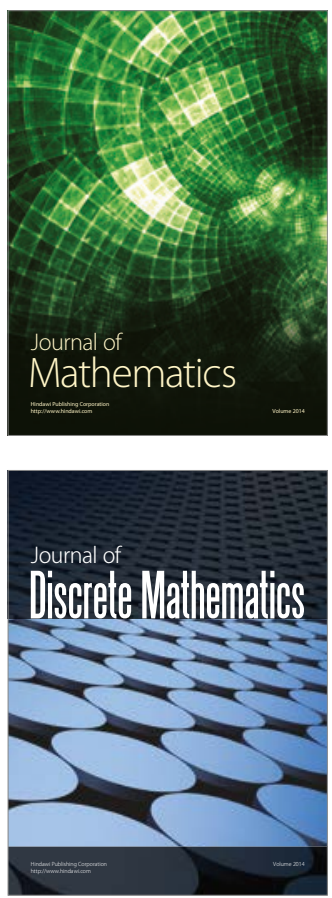

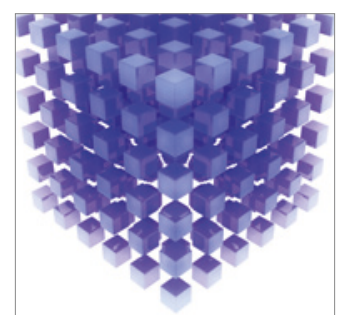

Mathematical Problems in Engineering
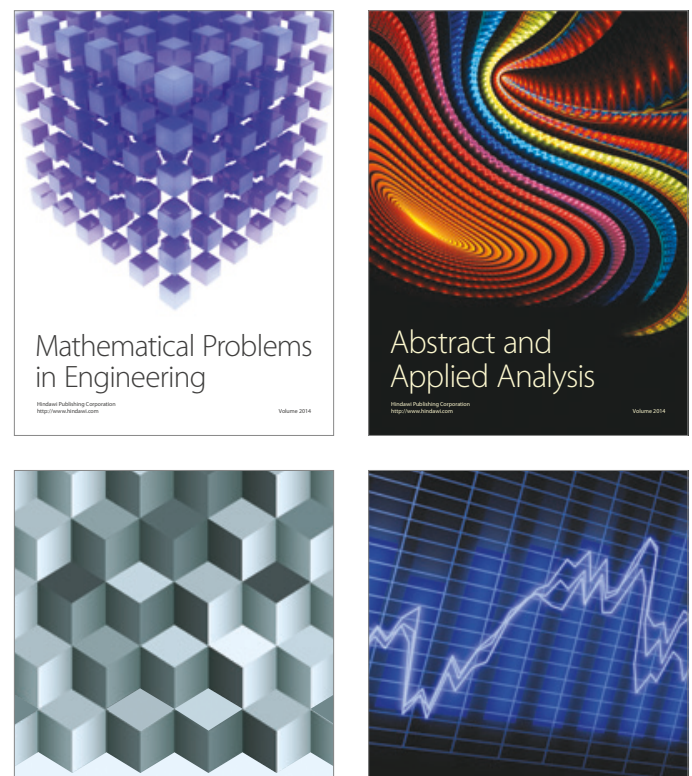

Journal of

Function Spaces

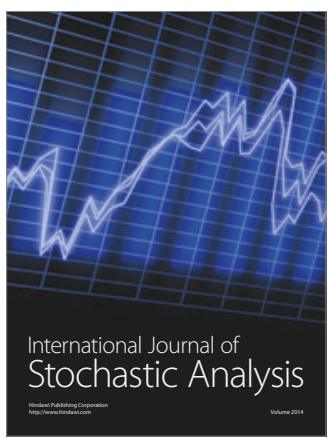

Probability and Statistics
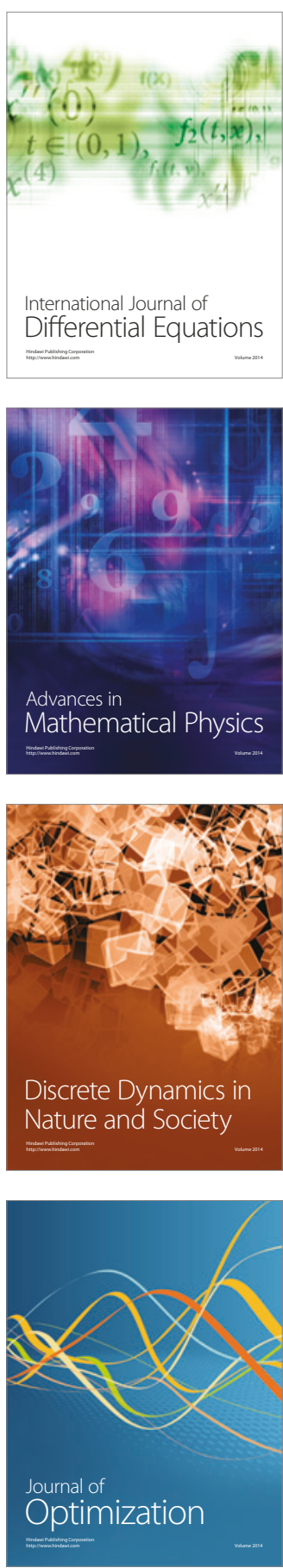